\title{
Pause Time Optimal Setting for AODV Protocol on RPGM Mobility Model in MANETs
}

\author{
Sayid Mohamed Abdule, Suhaidi Hassan, Osman Ghazali, Mohammed M. Kadhum \\ InterNetWorks Research Group \\ College of Arts and Sciences \\ University Utara Malaysia \\ 06010 UUM Sintok, Malaysia \\ sayidabdule@internetworks.my|\{ suhaidi|osman|kadhum\}@uum.edu.my
}

\begin{abstract}
For the last few years, a number of routing protocols have been proposed and implemented for wireless mobile Ad hoc network. The motivation behind this paper is to discover and study the pause time effects on Ad hoc on Demand Distance Vector (AODV) protocol and find out the node pause time optimal setting for this protocol where Reference Point Group Mobility (RPGM) model uses as a reference model. In order to come across the best performance of a particular routing protocol, there a need to examine a number of parameters with different performance and analyze the optimal setting of that protocol and its network configuration environment. This experiment, the speed is fixed with $20 \mathrm{~ms}$ in all scenarios while the pause time is varying from scenario to another to observe the optimal setting of the pause time on protocol's performance in this configuration. The outcome of the experiment are analyzed with different parameters such as varying number of nodes, increasing connections, increasing pause time and discussed the effects of the pause time. The results have shown that the value of the pause time can be affecting the performance of the protocol. In the experiment, we found that the lower pause time give better performance of the protocol. However, this paper is a part of ongoing research on AODV protocol in link failure. Thus, it is important to figure out the factors which can be involved the performance of the protocol.
\end{abstract}

Keyword-MANETs, AODV, pause time, optimal settin, RPGM.

\section{INTRODUCTION}

A mobile ad hoc network is a number of devices that communicates each other without any central administration and each of them acts as a router as it receives packet and forwards if it is not the destination. Actually Mobile Ad hoc Networks (MANETs) is a wireless communication and it uses a wireless interface to send and receives packet data, but in different way from infrastructure wireless. However, Mobility models are important building blocks in wireless networks and ad hoc developers can choose from a range of models that have been developed in the wireless both infrastructure less and infrastructure [1]. Ad hoc networks can be considered as a flexible application and uses for some special occasions that are inconvenient or unable to infrastructure-network. These applications which can be used in this communication are as follows: disaster aid, police operations in particular areas, group conferences, rescue operations, military deployment in aggressive environment etc. In practically, there are many applications that can be applied by Ad hoc networks. Therefore, the Mobile Ad hoc Networks (MANETs) are of much interest to network developers both public and the privet sectors because of its potential applicable to establish an easy communication network in any situation that involves both emergencies and normal applications [2]. There are a number of mobility models which have been widely used for evaluating the performance of relevant protocols or algorithms in traditional Ad hoc networks. Among of these models are Random Waypoint (RW) model, Reference Point Group Mobility (RPGM) model, Freeway Mobility (FW) model, Manhattan Grid (MG) model, Gauss-Markov (GM) and many others [3]. In this paper, is used the Reference Point Group Mobility (RPGM) to generate the mobility scenarios to evaluate the performance of the protocol in this particular configuration. Most of MANETs simulations are used Random Waypoint (RW) as a reference mobility model [4], [5]. Thus, there is a need to provide additional mobility models in order to examine many different MANET applications.

\section{MOBILITY MODELS}

MANETs, mobile nodes move from point to another point. The main role of mobility models is to emulate this movement of real mobile nodes. Mobility models are based on setting out different parameters related to node movement in order to evaluate in different metrics performance in different routing protocols. These parameters are for examples the starting location, the nodes movement direction, velocity range, speed changes over time and so on. Mobility models can be categorized into two types and they are as follows [6]:

$$
\begin{aligned}
& \text { •Entity Models } \\
& \text { •Group Models }
\end{aligned}
$$

When the mobile nodes are going to apply Entity models, the movements of the mobile nodes are completely independently from each other. In contrary, when the mobile nodes are using in group models, the mobile nodes are dependent on each other and follow predefined leader node. 
One of the prominent group models is Reference Point Group Mobility (RPGM) model which represents the random motion of a group of mobile nodes and their random individual motion within the group. In this model, the group motion behavior is determined by a logical group center where all group members have to follow that group leader. The individual mobile nodes movements or the entity mobility models should be specified the way of the movement of the individual mobile nodes. These movements are independent and each every of mobile nodes can move to its way randomly with randomly velocity. The principle of that the logical group center for RPGM model is to guide group of nodes continuously calculating group motion vector $\overrightarrow{\mathrm{GM}}$ in order to define; speeds and directions for mobile nodes. However, once the updated reference point $\mathrm{RP}(\mathrm{t}+1)$ has been updated they are combined with random motion vector $\overrightarrow{\mathrm{RM}}$ values to represent the random motion of each mobile node around its reference point [7].

\section{ROUTING PROTOCOLS}

In Ad hoc networks, entire network nodes are needed to perform as routing functions. To manage with the dynamic nature of the topology of Ad hoc networks, several routing protocols have been proposed. Taking into consideration of procedures for route establishment and update, MANET routing protocols can be categorized into three types:

\section{-Proactive \\ -Reactive and \\ -Hybrid protocols}

Proactive (table-driven) protocols maintain the routing information consistently up-to-date from each node to every other node in the network. The main function of proactive routing protocol maintains its table in order to store routing information. Up on changing in the network topology caused by anything just need to be reflected to this table and propagate the updating information throughout the network. Reactive (on demand) protocols are based on source-initiated on-demand reactive routing. The reactive routing protocol initiates routes only when mobile node requires a route to a destination. This method works like that the node broadcasts a route discovery initiative on demand and finishes when the destination is fund. Hybrid protocols are combination of proactive and reactive protocols. An example for proactive protocol is DSDV. This protocol routing protocol, all the possible destinations and the number of hops in the network are stored in a table. Because of ad hoc networks instabilities the table updating may change extremely dynamically and updating advertisements might be caused more network congestion and increases network routing overhead as well. AODV is a reactive protocol that improves the DSDV in the sense of minimizing the number of extremely advertisements and an unnecessary hello messages by creating routes on a demand mechanism [7]. Three main of Route Request process for AODV protocol are:

$$
\begin{aligned}
& \text {-Route request (RREQ) } \\
& \text {-Route reply (RREP) } \\
& \text {-Route error (RERR) }
\end{aligned}
$$

Figure 1shows that the source broadcasts a route request to its entire neighbors and each of these neighbors forwards the request until it reaches at the destination. Figure 2 illustrates the reverse route propagating to source. When the destination receives a routing packet from the source, it replies the shortest path and the source will use this path to send data packets. Figure 3 presents a route error. After the path between source and destination is established, the data is transferring. But due to the Ad hoc network characteristic of rapid topology change, the link between the source and the destination breaks. When such accident happens in Ad hoc networks using AODV routing protocol the current node prepares an error message propagate to the source. In this case the current node is the node 2 in Figure 3 and the link between node 2 and node 3 is broken, thus the node 2 prepared an error message and sent to the source.[8]
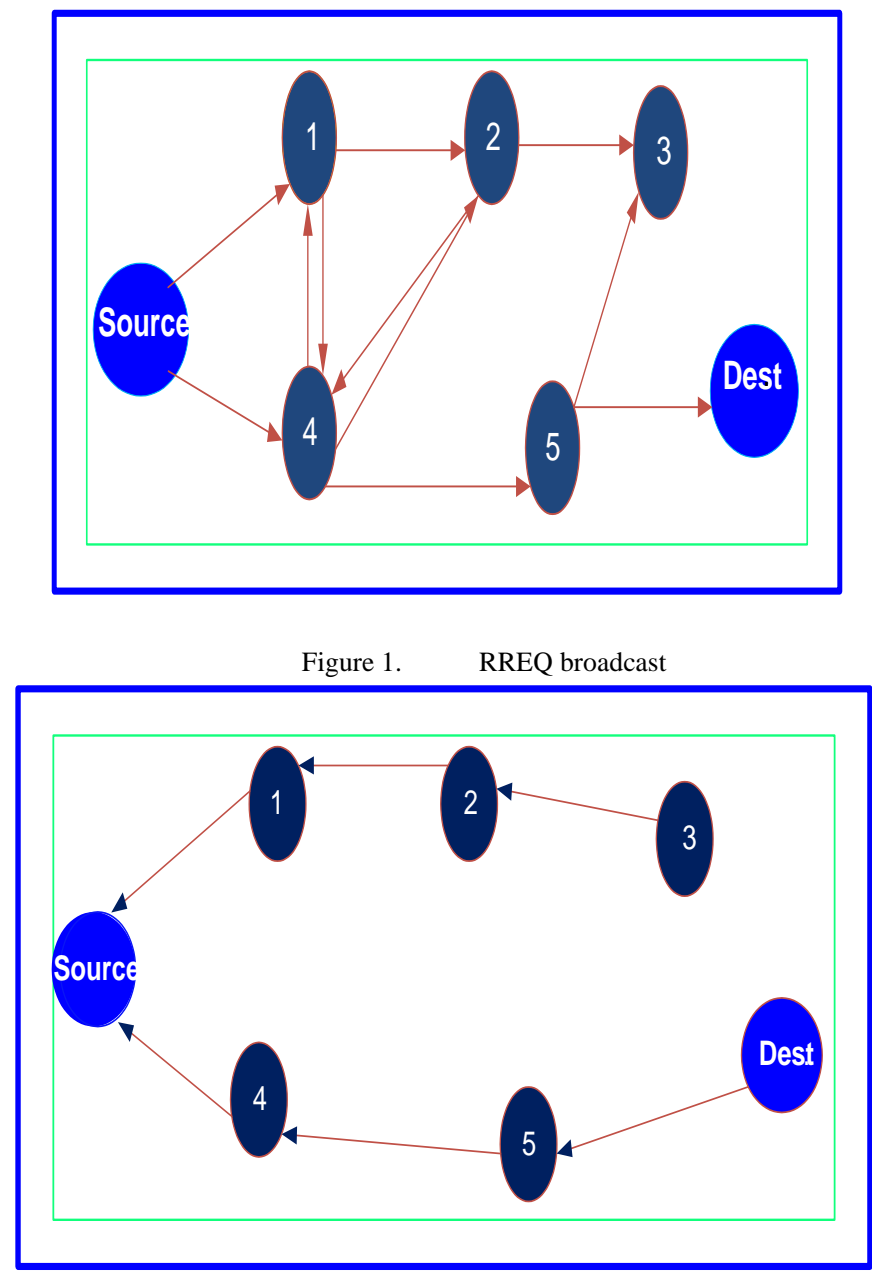

Figure 2. RREP 


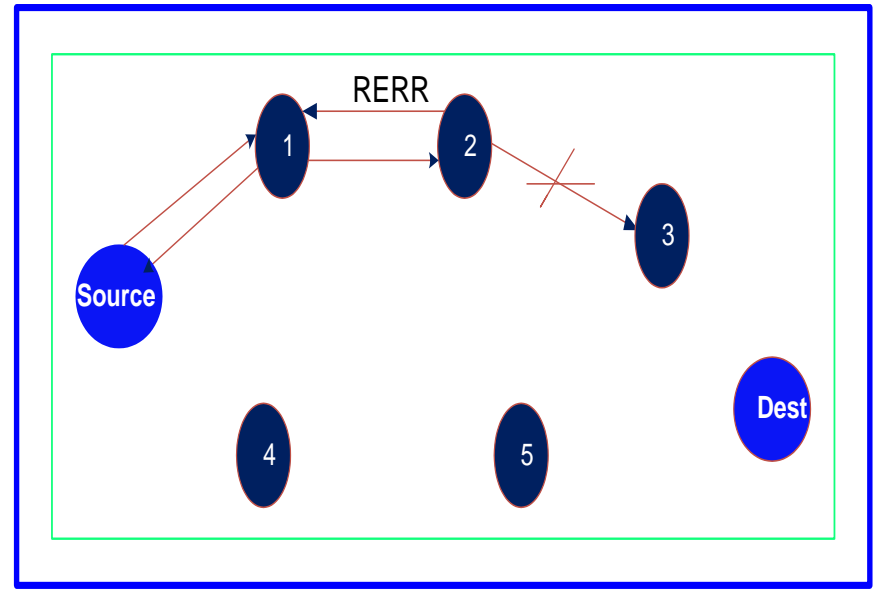

Figure 3. Route Failure

\section{NETWORK CONFIGURATION}

In this section will be analyzed the protocol performance optimal setting using Reference Point Group Mobility model (RPGM) and set up network configuration. These experiments have been conducted to finalize the maximum pause time optimal setting for protocal performance. In this set of simulations, our intention is to investigate the protocol's performance under RPGM when the pause time increases. The results present the performance of the protocol is very much depending on pause time value. From that point of view it is very important to run several simulations with different parameters in order to find out the suitable value of pause time for the Protocol. Network set up, we increase the pause time from 5 up to $40 \mathrm{sec} 40$ and keep all other parameters unchanged in first scenario. Keep in mind that for each scenario we keep running five times and these five times the pause time increases $(5,10,20,30 \& 40 \mathrm{sec})$ while all other parameters are fixed. In the 2 nd, third and fourth scenarios, we increase the number of nodes by double (from 10 to 20 nodes, 20 to 40 and 40 to 80 ) and number of connection is also increases as well $(4,8,30 \& 40$ connections) respectively and of course for each scenario the pause time increases while the rest of network parameters unchanged. The following tables are indicated the scenarios that have been tested in this experiment.

The following tables are four main scenarios and each of them contains five sub-scenarios. Each of these sub-scenarios, the pause time is varying while the rest of the parameters are constant or unchanged. When the first scenario has tested the five sub-scenarios with varying pause time, we continue, the second main scenario with increasing the number of nodes and number of connection with 20 nodes and 8 connections respectively as the table1-2 has shown. This second scenario has also tested same as tested the scenario one with five subscenarios except that increasing the number of nodes and connections and of course the pause time is also varying. Subsequently the third and fourth scenarios keep carrying on with increasing the number of nodes and connections 40 nodes and 80 nodes with 30 and 40 connections respectively as the table1-3 and table1-4 are indicated.
TABLE I. VARYING PAUSE TIME (RPGM-MODEL)

\begin{tabular}{||c||c||}
\hline \multicolumn{1}{||c||}{ Parameters } & Values \\
\hline Simulation & Ns-2 \\
\hline \hline Protocol & AODV \\
\hline \hline Movement Model & RPGM \\
\hline Traffic source & Constant Bit rate (CBR) \\
\hline Simulation area & $1000 \mathrm{~m} \times 1000 \mathrm{~m}$ \\
\hline \hline Simulation Time & $200 \mathrm{sec}$ \\
\hline \hline \# of nodes & 10 \\
\hline \hline Pause time & $5,10,20,30 \& 40$ \\
\hline \hline Nodes speed & $20 \mathrm{~m} / \mathrm{s}$ \\
\hline \hline \# node of sourceS & 4 \\
\hline
\end{tabular}

TABLE II. VARYING PAUSE TIME (RPGM-MODEL)

\begin{tabular}{||c||c||}
\hline \multicolumn{1}{||c||}{ Parameters } & Values \\
\hline Simulation & Ns-2 \\
\hline Protocol & AODV \\
\hline \hline Movement Model & RPGM \\
\hline \hline Traffic source & Constant Bit rate (CBR) \\
\hline Simulation area & $1000 \mathrm{~m} \times 1000 \mathrm{~m}$ \\
\hline Simulation Time & $200 \mathrm{sec}$ \\
\hline \# of nodes & 20 \\
\hline \hline Pause time & $5,10,20,30 \& 40$ \\
\hline Nodes speed & $20 \mathrm{~m} / \mathrm{s}$ \\
\hline \hline \# node of sources & 8 \\
\hline \hline
\end{tabular}

TABLE III. VARYING PAUSE TIME (RPGM-MODEL)

\begin{tabular}{||c|c||}
\hline Parameters & Values \\
\hline \hline Simulation & Ns-2 \\
\hline Protocol & AODV \\
\hline Movement Model & RPGM \\
\hline Traffic source & Constant Bit rate (CBR) \\
\hline Simulation area & $1000 \mathrm{~m} \times 1000 \mathrm{~m}$ \\
\hline Simulation Time & $200 \mathrm{sec}$ \\
\hline
\end{tabular}




\begin{tabular}{|c|c|}
\hline \hline \# of nodes & 40 \\
\hline \hline Pause time & $5,10,20,30 \& 40$ \\
\hline \hline Nodes speed & $20 \mathrm{~m} / \mathrm{s}$ \\
\hline \hline \# node of sources & 30 \\
\hline
\end{tabular}

TABLE IV. VARYING PAUSE TIME (RPGM-MODEL)

\begin{tabular}{||c|c||}
\hline \multicolumn{1}{||c|}{ Parameters } & Values \\
\hline \hline Simulation & Ns-2 \\
\hline \hline Protocol & AODV \\
\hline \hline Movement Model & RPGM \\
\hline \hline Traffic source & $1000 \mathrm{~m} \mathrm{x} \mathrm{1000} \mathrm{m}$ \\
\hline \hline Simulation area & $200 \mathrm{sec}$ \\
\hline \hline Simulation Time & 80 \\
\hline \hline \# of nodes & $5,10,20,30 \& 40$ \\
\hline \hline Pause time & $20 \mathrm{~m} / \mathrm{s}$ \\
\hline \hline Nodes speed & 40 \\
\hline \hline \# node of sources & \\
\hline \hline
\end{tabular}

\section{PERformance Metrics}

As RFC 2501 described, a number of performance metrics that can be used for evaluating the performance of a routing protocol for MANETs are four metrics. In addition, AODV developers were used these four metrics [9]. Thus, in this paper, we follow the general ideas described in RFC 2501 and we used similar metrics such as Packet Delivery Ratio, Average End-to-End Delay, Normalized Routing Load and Routing Overhead (Normalized MAC Load). All these performance metrics are important, but the packet delivery ratio and average end-to-end delay are most important for best-effort traffic. It does not mean that the other metrics are meaningless, but it means that the two first metrics have high priority than others. However, the normalized routing load is important as it will be used to evaluate the efficiency of the routing protocol. In order to calculate the Packet Delivery Ratio, we collect all received data packets which are delivered at the destination node and sum together than divide the total Data packets sent by source associated with the agent type AGT. As proactive protocols are normaly expected to have a higher control overhead than reactive protocols, the TCP data traffic application can cause additional packet loss and network congestion at the intermediate nodes thus we used CBR (continuous bit -rate) as data traffic generation in this paper. Calculating Average End-to-end delay, At the destination side we summarize the all receive packets(RecvPkts) then store the transmission time for each successful data and extracts the receiving time of that packet. These times are included all possible delays in the network such as broadcasting latency, the intermediate nodes retransmission delay, processing delay, queuing delay and propagation delay. Based on this information we calculate the end-to-end delay to summarize all those delays and divide by the number of RecvPkts. Normalized MAC Load(NML), can be define as the fraction of all control packets (routing control packets, included Request-To-Send (RTS) which means a request signal to next neighbor asking for if he can be ready to receive his request, Clear-To-Send (CTS) which means an acceptance for that request, Address Resolution Protocol (ARP) requests and replies, and MAC ACKs (incase using TCP ) divide the total number of received data packets. The normalized routing load (NRL), this metric is different from Normalized MAC load (NML) and sometimes is difficult to distinguish each other. The NRL concerns only received data packets at the destination while NML take into the account all establishing process for control packets in routing level not the agent (AGT) level. The way of calculation of NRL is defined as the fraction of all routing control packets sent by all nodes divide the number of received data packets at the destination nodes.

\section{RESUltS AND ANALYZES}

\section{A. Routing Overhead Using Rpgm}

This metric presents how the network enquiry packets control for intermediate nodes is huge. To evaluate the network efficient, the routing overhead or Normalized MAC Load plays a significant roll. Thus it is vital for this paper to figure out the vectors which can be involved the increasing of the network routing overhead. However, minimizing the network overhead is maximizing the network resources utilization with better performance. Figure 4 presents the experiment result for routing overhead. The Figure 4 shows that the overhead is direct proportional to pause time. It indicates that the overhead increases while the pause time is increased. This experiment tested five different values of pause time, namely $5,10,20,30 \& 40 \mathrm{sec}$. The figure 4 illustrated four scenarios and each has a different value than others. These scenarios have been analyzed one by one as we get a better overview.

Look at the scenario one, when the pause time is $5 \mathrm{sec}$ the overhead is 54 , when we increase the pause time to $10 \mathrm{sec}$ the overhead became 186, then 330,408 and finally when the pause time increase up to $40 \mathrm{sec}$ the overhead is worst. Second scenario, this scenario is totally different from previous scenario because the overhead started 442 while scenario one the overhead started 54, because of varying node density in the network. But the principle is stil same because when the pause time is increased the overhead is also increased. The Scenarios three and four are some as these two scenarios, increase the pause time, the overhead goes up. So, seeing the scenarios in the Figure 4 the overhead is definatly proportional to pause time and obviously the overhead will be low when the pause time is small. Thus, this experiment shows that choosing a smaller pause time, gives the optimal setting for node pause time and indicates that the average performance of AODV protocol is when the node pause time is between $5 \mathrm{sec}$ and $10 \mathrm{sec}$. 


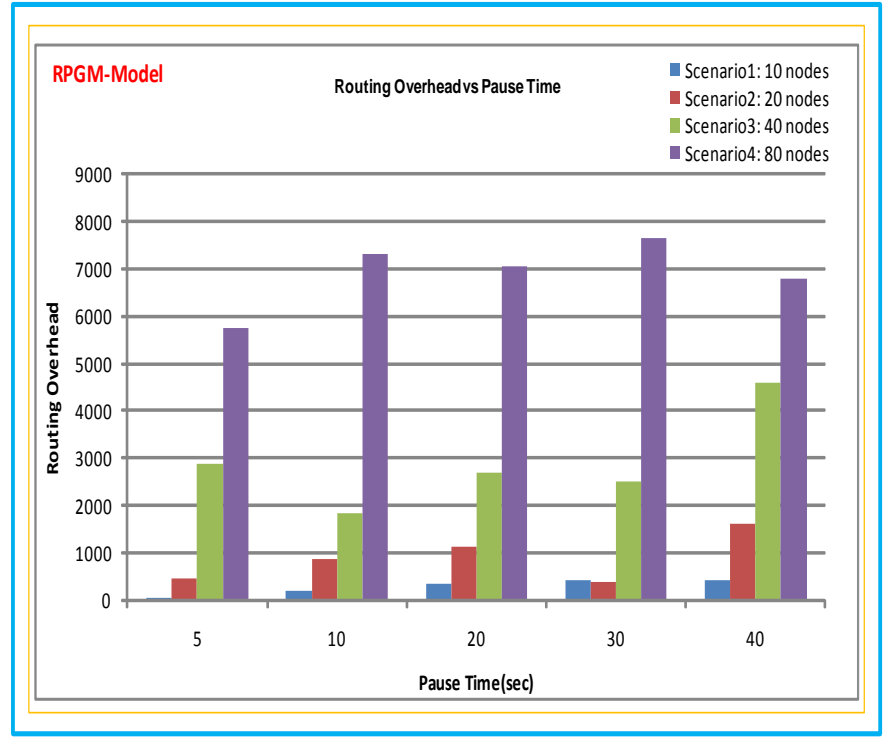

Figure 4. Routing overhead using

\section{A. Packet Delivery Fraction Using RPGM}

Figure 5 shows the result of Packet Delivery Fraction (PDF). This part of analyzing is important as it describes the rate of packets drop as well as it affects the overall network throughput that the network can support. Figure 5 shows that the packets delivery ratios is mostly constant whether the the pause time increases or not. Only the scenario one shows little bit flactuation where the pause time for 10 and 20 are $95.06 \%$ and $93.35 \%$ respectively. Scenarios two, three and four have shown the best result and most values are between $98 \%$ and 99\%. Nonetheless, the overall results for the packets delivery ratios based on RPGM model indicate that the packet delivery is very smooth for all scenarios.

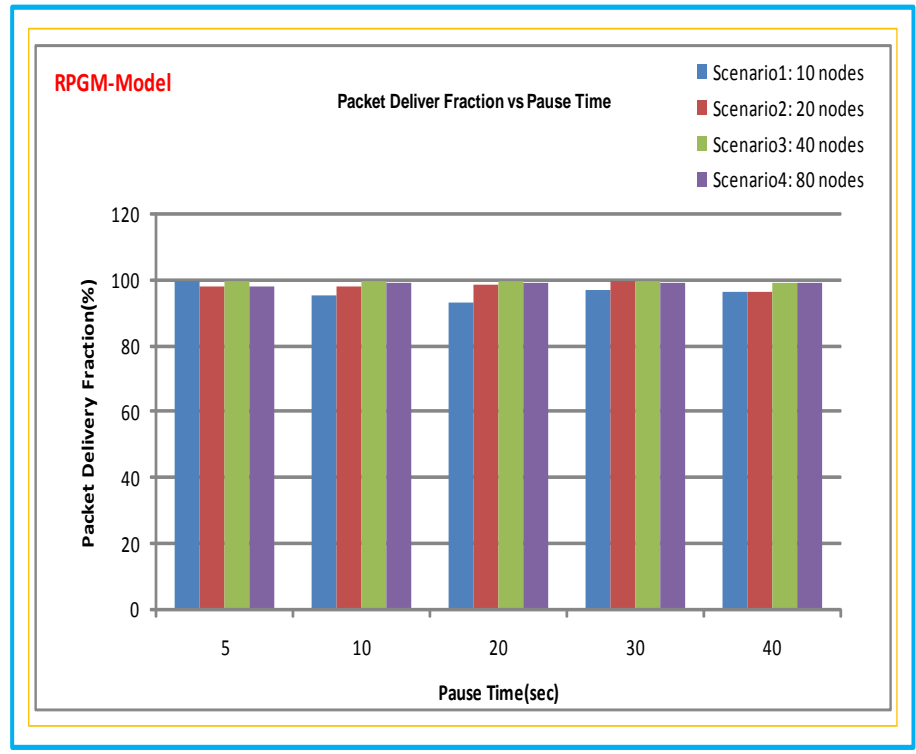

Figure 5. Packet Delivery Fractions

\section{B. Normalizing Routing Load Using RPGM}

Figure 6 shows the simulation for Normalized Routing Load with the RPGM model. The objective of this part of analyzing is to investigate the impact of varying pause time on the normalizing route load in the network. The Figure 6 shows the scenarios of the simulation for normalizing routing load. It shows that as the pause time increases, the normalized routing load increases. For instance, when the pause time is 5 in scenario one the normalized routing load shows $0.05 \%$. Then we increased the pause time up to $10,20,30$ and 40 in same scenario, the results show $0.18 \%, 0.33 \%, 0.39 \%$ and $0.40 \%$ respectively. This analyzing indicates that as the pause time goes up, the result gets worest which meas that the network becomes more and more congested in terms of traffic load.

The rest of scenarios are almost same as previous scenario (scenario one) and show when ever the pause time increases the result of NRL increases, but scenarios two and three have shown when the pause time is 30 and 10 the result do not follow the previous results which were increasing when the pause time increases instead they decrease in this time as can be seen in the Figure 6 the results show $0.16 \%$ and $0.34 \%$. Since the most of results are shown the same direction when the pause time is increased, we can assume these two cases are accidently happen because of the ad hoc network behavior is very changeable. NRL represents the number of routing packets transmitted per data packet delivered at the destination, as it to evaluate the efficient of protocol's performance. However, in this experiment indicates that when the pause time is between 5 and $10 \mathrm{sec}$ is the optimal setting for AODV protocol when using RPGM model.

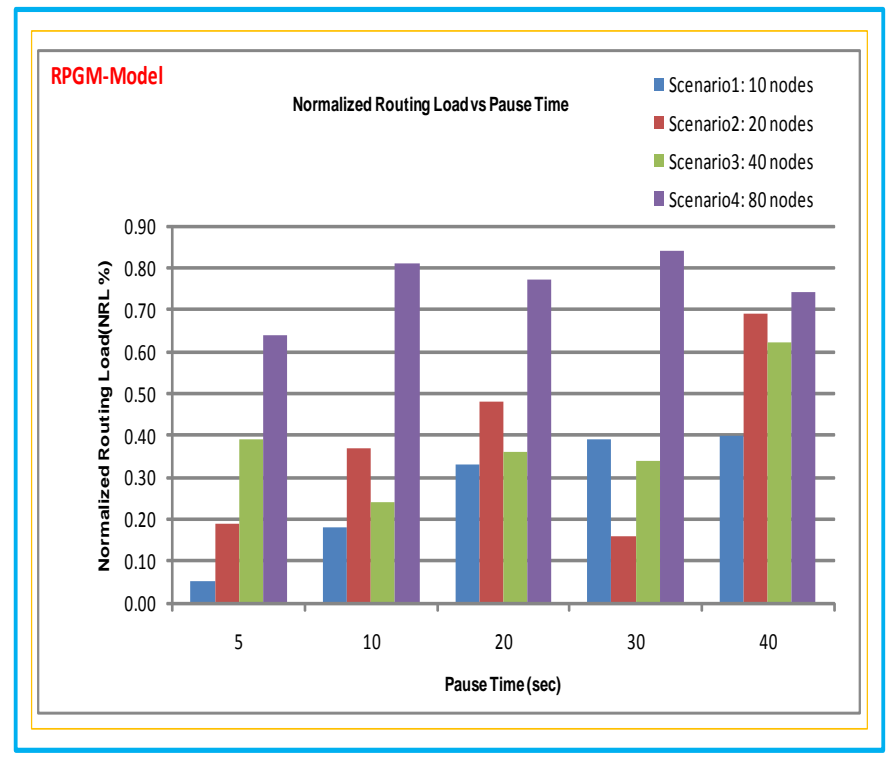

Figure 6. Normalized Routing Load

\section{Result for Average end to End Delay}

Figure 7 illustrates average end to end delay result for RPGM model. The Figure 7 shows that the delay of scenario one where the pause times are varying from 5, 10, 20, 30 and $40 \mathrm{sec}$. It shows when the the pause time is 30 the average end 
to end delay is $11.81 \%$. So, the pause time is increased to10 and the result for this scenario is also increased up to 113.23 $\%$. Then we increased the pause time up to 20 , so the delay is also increased up to $204.43 \%$. Again, the pause time is increased from 20 to 30 but as the figure 7 indicates in scenario one the result decreased $178.44 \%$. Finally, we increased the pause time up to 40 and result decreased as well $135.22 \%$. It seems that the average end to end delay is not that much affected by the varying of pause time. We expected that the lower pause time will have a better performance average end to end delay than the higher pause time according to other parameters results in Figures 4, $5 \& 6$. Normally, the network parameters are depending on one other, and usually there is no one parameter that cannot be affected by another vector in order to be independent completely. However, the explanation lies in that the delay can be independent to some extent.

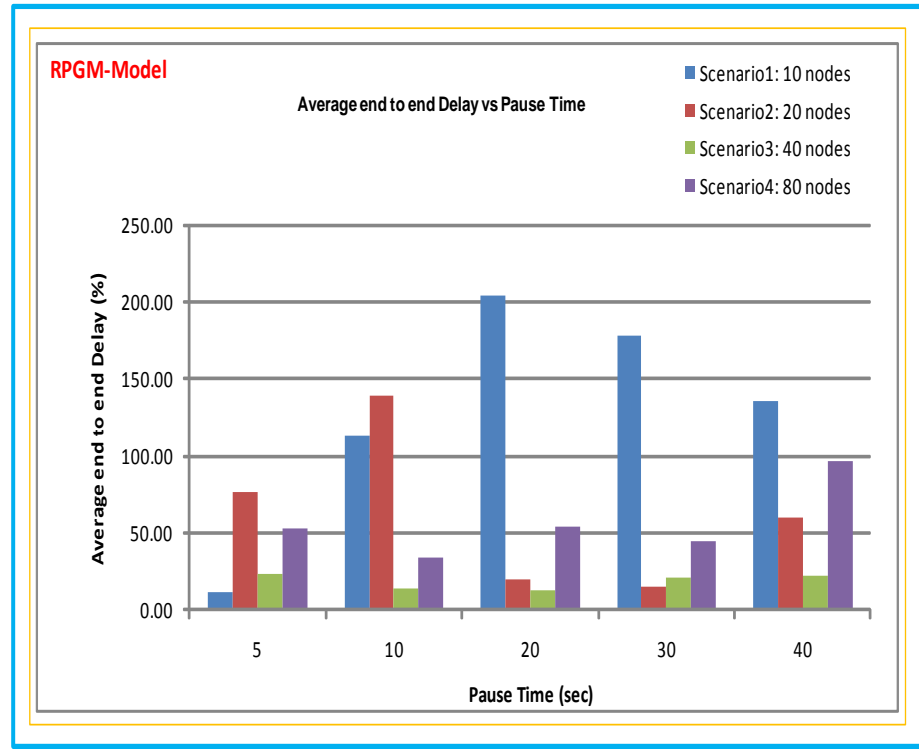

Figure 7. Average end to end Delay

\section{Packet Loss}

The Figure 8 presents data packets drop. In theoretical, the reactive routing protocols and proactive routing protocols differ drastically in the fact that they belong to two different routing families. Reactive routing protocol generates or flood packets on demand only, in order to reduce routing loads. Proactive protocol frequently updates the routing tables regardless of need. This proactive periodically message exchanging, causes tremendous routing overhead. These overhead can lead dropped data packets in the network. In contrary, the proactive routing protocols are expected less routing overhead because there is no need periodically information exchange to update the tables. This minimizing of information exchange for reactive routing protocol is an advantage for reactive routing protocol and will lead minimizing dropped data packets. However, the proactive routing protocols have also different behavior. AODV has more overhead than other reactive routing protocols caused by AODV route query and routing overhead is proportional to the number of route queries. For instance, the source node for
AODV will send a RREQ message if it does not know the route to the destination. The source waits for a while, if the source sends the second transmission of the RREQ message and does not receive RREP message within a time interval, it will drop the first packet in the queue and repeats the same procedure for the second data packet in the queue.

In addition, if one of the forwarding nodes cannot success to find a valid route to the destination will drop all data packets from its queue. Here we are going to examine the pause time optimal setting for AODV protocol in packet drop using RPGM as a model. The results have shown that the dropped data packets in this experiment are very reasonable and much better than expected results. Because, as above mentioned the AODV protocol has heavy routing overhead which can cause a significant drop data packets. The Figure 8 shows that dropped data packets for the scenario one are 2, 59, $90,55 \& 59$. First experiment in this scenario has only 2 packets lost while the second third, fourth and fifth experiments have 59, 90, 55 and 59 packets lost respectively. Drops for scenario two are $49,68,83, \& 158$ while third scenario results are $81,83,61 \& 170$ and 167 . However, the three first scenarios optimal setting is when the pause times are 5 and 10 .

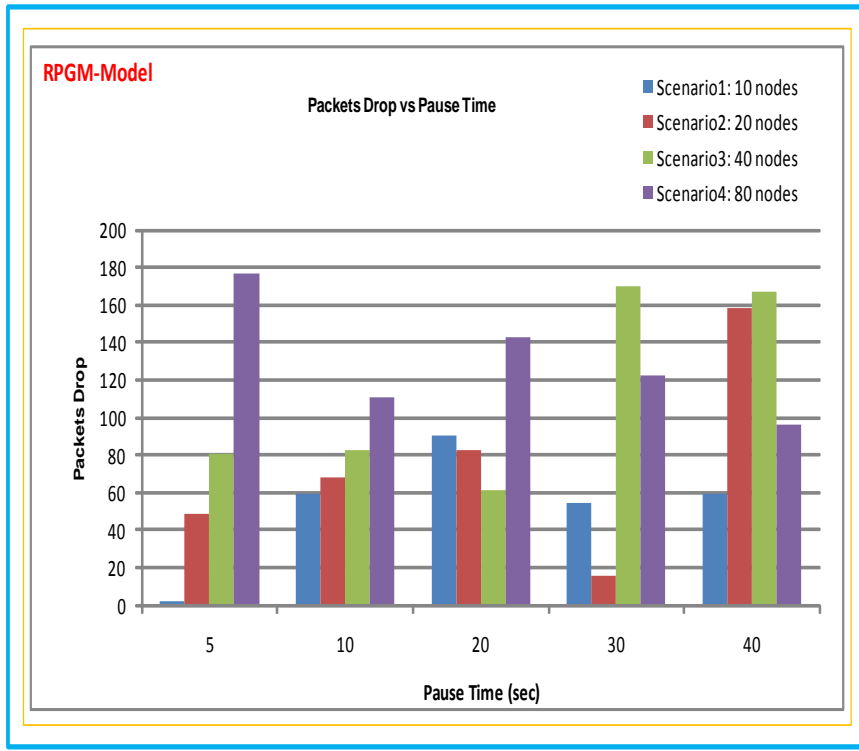

Figure 8. Packet drop

\section{CONCLUSION}

This paper, we have conducted several experiments and analyzed the effects of pause time varying to evaluate the performance of AODV protocol based on RPGM as a reference model. The simulation results evaluated the performance of the routing protocol with regard five performance metrics such as Packet Delivery Ratio, Average end to end delay, Packet Drop, Routing overhead and Normalized routing load. The experiment results for all scenarios present that the low pause time, the high performance of the protocol. The experimentation also suggests that several parameters such as traffic patterns, node 
density are also affect the routing performance and need to be investigated with various scenarios. Further study also needs to be done with additional analysis with different mobility models.

\section{REFERENCES}

[1] Yang Huan, Jiang Hong, Liu Lei "Performance Analysis of Mobility Models in Sparse Ad-hoc Networks" School of Information Engineering, Southwest University of Science and Technology, Mian Yang 621010,P.R.China, Proceedings of the 27th Chinese Control Conference July 16-18, 2008, Kunming, Yunnan, China,2008.

[2] Tariq M M B,Ammar M and Zegura E. "Message Ferry Route Design for Sparse Ad hoc Networks with Mobile Nodes", In Proc. The 6th ACM International Symposium on Mobile Ad Hoc Networking and Computing (Mobi-Hoc'06), 2006.

[3] C.P.Agrawal et al "Evaluation of Varrying Mobility Models \& Network Loads on DSDV Protocol of MANETs" Raipur, India, International Journal on Computer Science and Engineering Vol.1(2), 2009, 40-46.

[4] S. Sesay et al., "Simulation Comparison of Four Wireless Ad Hoc Routing Protocols",Information Technology Journal 3(3), 2004, pp:219226.

[5] S. Shah et al, "Performance Evaluation of Ad Hoc Routing Protocols Using NS2 Simulation",Conf. of Mobile and Pervasive Computing, 2008.

[6] T. Camp et al., "A Survey of Mobility Models for Ad Hoc Network Research", Wireless Comm. \& Mobile Computing: Special issue on Mobile Ad Hoc Networking: Research, Trends and Applications, vol. 2, pp. 483-502, 2002.

[7] Valentina Timcenko, Mirjana Stojanovic, Slavica Bostjancic Rakas,"MANET Routing Protocols vs. Mobility Models: Performance Analysis and Comparison" Proceedings of the 9th WSEAS International Conference on Applied Informatics and Communications (AIC '09).

[8] Jungkeun Yoon, Mingyan Liu, Brian Noble "Random Waypoint Considered Harmful" Electrical Engineering and Computer Science Department University of Michigan Ann Arbor, Michigan 48109-2122, IEEE INFOCOM 2003.

[9] Das, S.R., Perkins, C.E. Royer, E.M.2000, "Performance comparison of two on-demand routing protocols for ad hoc networks ", INFOCOM 2000. Nineteenth Annual Joint Conference of the IEEE Computer and Communications Societies. Proceedings IEEE Volume 1, pg. $3-12$, 26-30 March 2000.

\section{AUTHORS PROFILE}

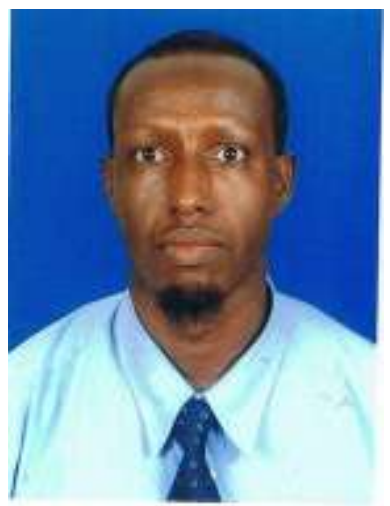

Sayid Mohamed Abdule, received his BSc degree in Computer Science from Agder University, Norway. And, his M.Sc degree in Satellite Communication focusing Quality of Service of VOIP over Satellite from the University Sains Malaysia (School of Computer Science), MALAYSIA. Sayid currently attached to the InterNetWorks Research Group at the UUM College of Arts and Sciences as a doctoral researcher. $\mathrm{He}$ is currently pursuing his $\mathrm{PhD}$ research in Ad-hoc Mobile networking. His current research interest is on Ad-hoc mobile network routing protocol.

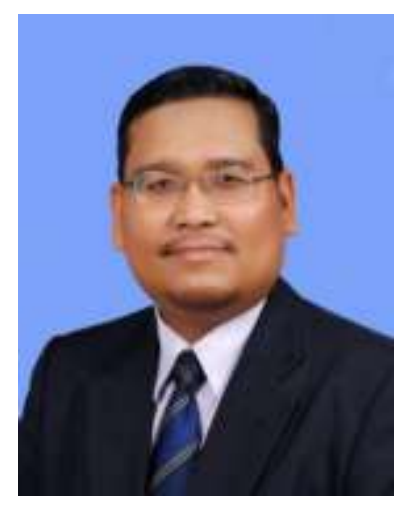

Associate Professor Dr. Suhaidi Hassan is currently the Assistant Vice Chancellor of the College of Arts and Sciences, Universiti Utara Malaysia (UUM). He is an associate professor in Computer Systems and Communication Networks and the former Dean of the Faculty of Information Technology, Universiti Utara Malaysia. Dr. Suhaidi Hassan received his BSc degree in Computer Science from Binghamton University, New York (USA) and his MS degree in Information Science (concentration in Telecommunications and Networks) from the University of Pittsburgh, Pennsylvania (USA). He received his $\mathrm{PhD}$ degree in computing (focusing in Networks Performance Engineering) from the University of Leeds in the United Kingdom. In 2006, he established the ITU-UUM Asia Pacific Centre of Excellence (ASP CoE) for Rural ICT Development, a human resource development initiative of the Geneva-based International Telecommunication Union (ITU) which serves as the focal point for all rural ICT development initiatives across Asia Pacific region by providing executive training programs, knowledge repositories, R\&D and consultancy activities. Dr. Suhaidi Hassan is a senior member of the Institute of Electrical and Electronic Engineers (IEEE) in which he actively involved in both the IEEE Communications and IEEE Computer societies. He has served as the Vice Chair (20032007) of the IEEE Malaysia Computer Society. He also serves as a technical committee for the Malaysian Research and Educational Network (MYREN) and as a Council Member of the Cisco Malaysia Network Academy.

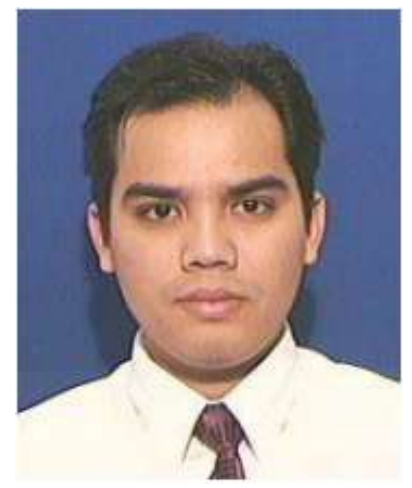

Osman Ghazali, Ph.D.

is a Senior Lecturer at Northern University of Malaysia (Universiti Utara Malaysia). He obtained his Bachelor and Master degrees in Information Technology in 1994 and 1996 from the Northern University of Malaysia. In 2004 he obtained his $\mathrm{PhD}$ specializing in Computer Network from Northern University of Malaysia. As an academician, his research interests include congestion control, quality of services, wired and wireless network, transport layered protocols and network layered protocols. His works have been published in international conferences, journals and won awards on research and innovation competition in national and international level. 


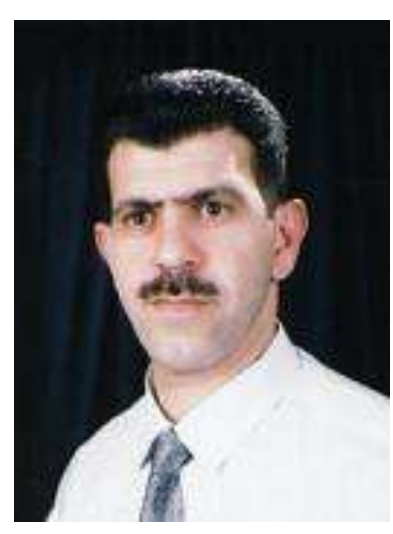

Mohammed M. Kadhum, Ph.D. is an assistant professor in the Graduate Department of Computer Science, Universiti Utara Malaysia (UUM) and is currently attached to the InterNetWorks Research Group at the UUM College of Arts and Sciences as a research advisor. He had completed his $\mathrm{PhD}$ research in computer networking at Universiti Utara Malaysia (UUM). His research interest is on Internet Congestion and QoS. He has been awarded with several medals for his outstanding research projects. His professional activity includes being positioned as Technical Program Chair for NetApps2008 and NetApps2010, a technical committee member for various well known journal and international conferences, a speaker for conferences, and a member of several science and technology societies. To date, he has published a number of papers including on well-known and influential international journals. 